\title{
Digital Table of Solar System
}

\author{
Lutvo Kurić \\ Institute of Economics, University of Sarajevo, Trg Oslobođenja 1, Sarajevo, \\ Bosnia and Herzegovina \\ E-mail address: lutvokuric@yahoo.com
}

\begin{abstract}
This paper discusses cyberinformation studies of the Solar System, in particular the identification of scientific terminology that could describe this phenomenon, that is, the study of Solar System, as well as the relationship between the astronomical language of Solar System and theoretical aspect of this system and cybernetics. The result of this research show that there is a matrix code for Solar System. It also shows that the coding system within the planetary language gives detailed information, not only on the planetary "record", but also on its structure, configuration and its various shapes. The issue of the existence of a planetary code and coding of the individual structural planets of this system are discussed. Is the planetary information characterized only by physical, or also by cyberinformation principles? The potential effects of physical and Orbital characteristics, as well as cybernetic and information principles, on the atronomical basis of Solar System are also investigated. This paper discusses new methods for developing astronomy technologies, in particular more advanced digital technology based on programming, cybernetics, and informational laws and systems, and how this new technology could be useful in astronomy, bioinformatics and other natural sciences.
\end{abstract}

Keywords: Digital table of Solar system; orbital code; perihelion and aphelion

\section{INTRODUCTION}

Solar System can be represented by two different forms, ie, a discrete form and a sequential form. In the discrete form, a Solar System is represented by a set of discrete codes or a multiple dimension vector. In the sequential form, a Solar System is represent by a astronomical and orbital characteristics of planets.

Therefore, the sequential form can naturally reflect all the information about the planets order and lenght of a Solar System. The key issue is whether we can develop a different discrete method of representing a Solar System that will allow accomodation of partial, if not all planets order information? Because a Solar System is usually represented by a series of planets should be assigned to these codes in order to optimally convert the planetary sequence order information into a series of numbers for the discrete form representation? 


\section{RESULTS}

The matrix mechanism of Solar System, the evolution of planets and especially, the astronomical evolution of cosmic language, have been analyzed by the application of cybernetic methods, information theory and system theory, respectively. The primary structure of a planetary systems is the exact specification of its planetary composition and the planetary bonds those planets.

\begin{tabular}{|c|c|c|c|c|c|c|c|}
\hline \multicolumn{8}{|c|}{ Perihelion and Aphelion planets of the Solar system } \\
\hline Merkur & Venus & Earth & Mars & Jupiter & Saturn & Uranus & Neptune \\
\hline Perihelion & Perihelion & Perihelion & Perihelion & Perihelion & Perihelion & Perihelion & Perihelion \\
\hline 46001200 & 107476259 & 147098290 & 206669000 & 740573600 & 1353572956 & 2748938461 & 4452940833 \\
\hline 1 & 2 & 3 & 4 & 5 & 6 & 7 & 8 \\
\hline $\begin{array}{c}\text { Merkur } \\
\text { Aphelion }\end{array}$ & $\begin{array}{c}\begin{array}{c}\text { Venus } \\
\text { Aphelion }\end{array} \\
\end{array}$ & $\begin{array}{c}\text { EARTH } \\
\text { Aphelion } \\
\end{array}$ & $\begin{array}{c}\begin{array}{c}\text { Mars } \\
\text { Aphelion }\end{array} \\
\end{array}$ & $\begin{array}{c}\text { Jupiter } \\
\text { Aphelion }\end{array}$ & $\begin{array}{c}\begin{array}{c}\text { Saturn } \\
\text { Aphelion }\end{array} \\
\end{array}$ & $\begin{array}{c}\begin{array}{c}\text { Uranus } \\
\text { Aphelion }\end{array} \\
\end{array}$ & $\begin{array}{c}\text { Neptune } \\
\text { Aphelion }\end{array}$ \\
\hline 69816900 & 108942109 & 152098232 & 249209300 & 816520800 & 1513325783 & 3004419704 & 4553946490 \\
\hline 1 & 2 & 3 & 4 & 5 & 6 & 7 & 8 \\
\hline
\end{tabular}

This text translates of prihelion and aphelion from a digital form.

\section{1. Algorithm}

\section{Input:}

\{46 $001200,69816900,107476259,108942109,147098290,152098232$, $206669000,249209300,740573600,816520800,1353572956$, $1513325783,2748938461,3004419704,4452940833,4553946490\}$
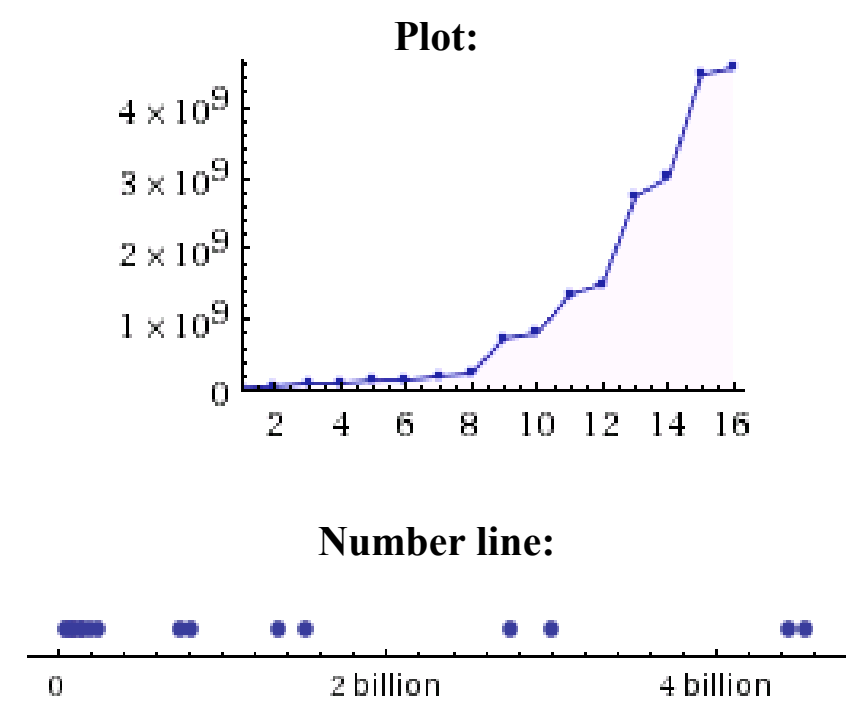


\section{Length of data:}

16 items

Total:

$46001200+69816900+\cdots+4452940833+4553946490=20271549917$

\section{Histogram:}

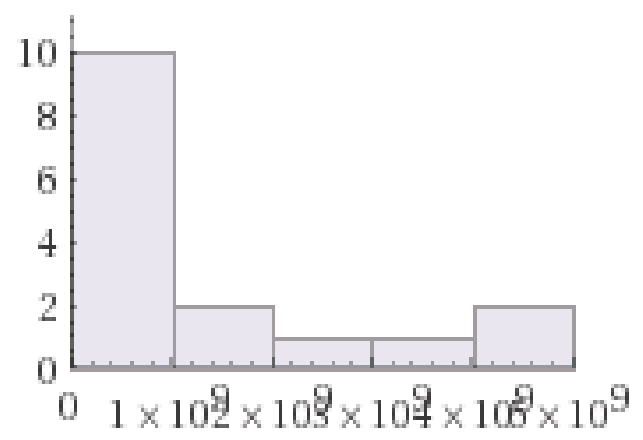

Statistics:

\begin{tabular}{|l|l|}
\hline mean & $1.267 \times 10^{9}$ \\
\hline median & $4.949 \times 10^{8}$ \\
\hline sample standard deviation & $1.57 \times 10^{9}$ \\
\hline
\end{tabular}

Box and whisker chart:

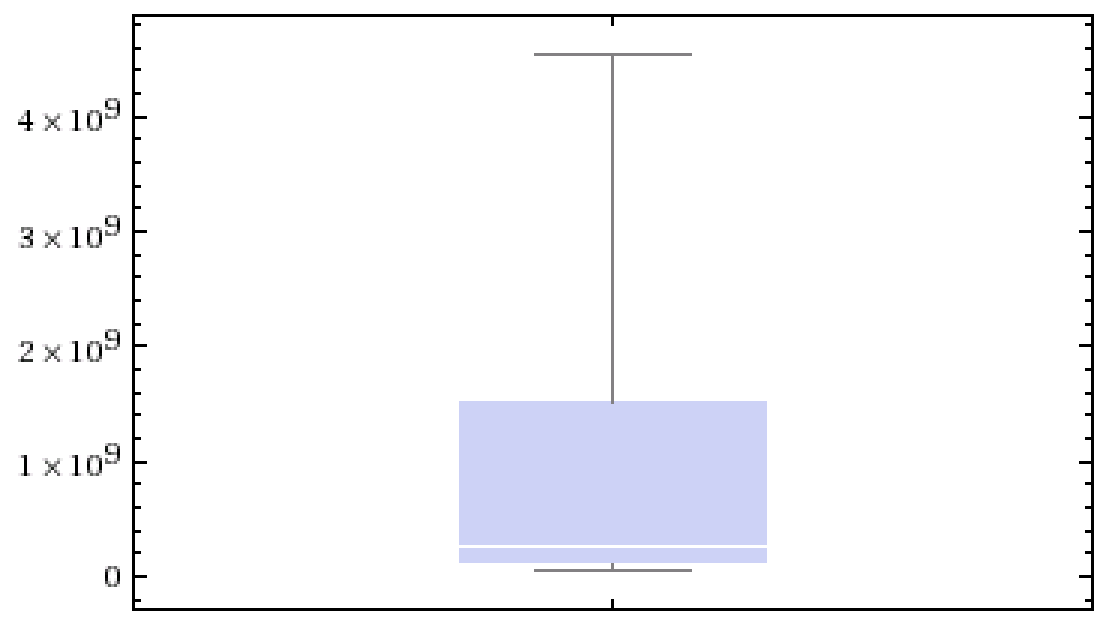




\section{Differences:}
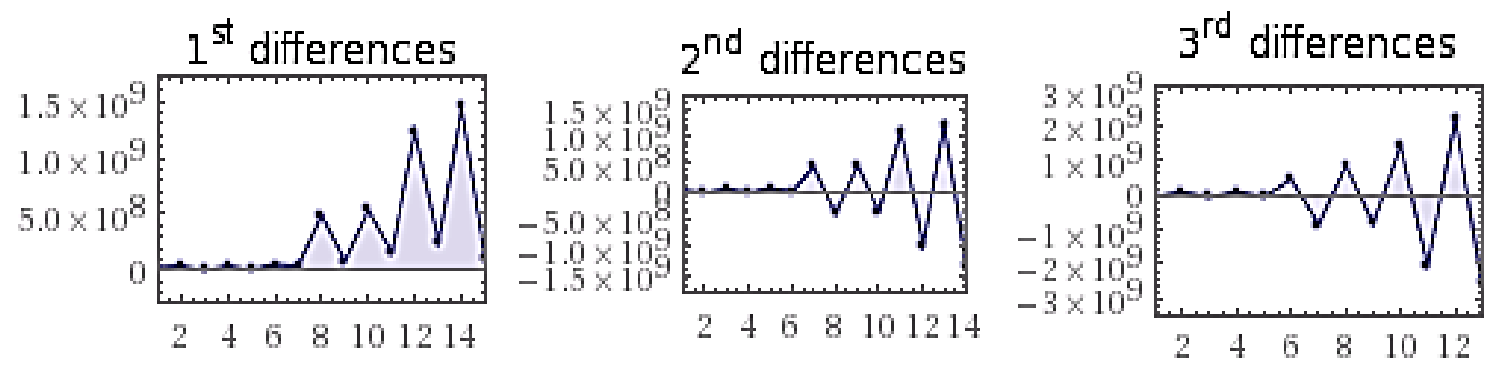

\section{Cumulative sums:}

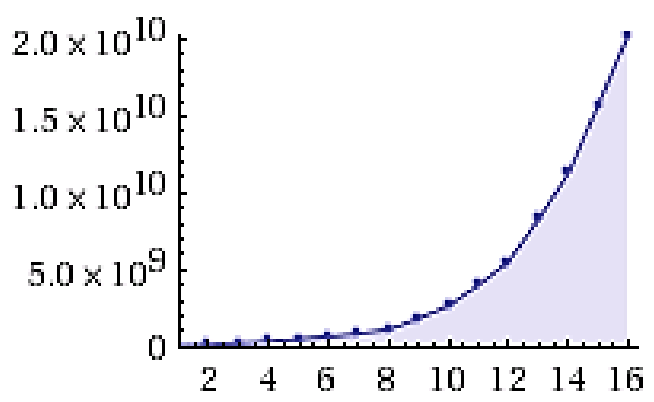

Succeessive ratios:

Using closest Wolfram|Alpha interpretation: ratios $\{46001200,69816900,107476259$, 108942109, 147098290, 152098232, 206669000, 249209300, 740573600, 816520800, $1353572956,1513325783,2748938461,3004419704,4452940833,4553946490\}$

\section{Input interpretation:}

$46001200: 69816900$ : $107476259: 108942109: 147098290: 152098232$ :

$206669000: 249209300: 740573600: 816520800: 1353572956$ :

$1513325783: 2748938461: 3004419704: 4452940833: 4553946490$

correlation $\{46001200,69816900,107476259,108942109,147098290,152098232$, 206669000, 249209300, 740573600, 816520800, 1353572956, 1513325783, 2748938461, $3004419704,4452940833,4553946490\}$

\section{Input:}

ListCorrelate[ [46 001 200, $69816900,107476259,108942$ 109, 147098290 ,

$152098232,206669000,249209300,740573600,816520800,1353572956$,

$1513325783,2748938461,3004419704,4452940833,4553946490\}$, $\{46001200,69816900,107476259,108942109,147098290,152098232$,

$206669000,249209300,740573600,816520800,1353572956$, $1513325783,2748938461,3004419704,4452940833,4553946490\}^{*},\{1\}$ 


\section{Result:}

$\{62667780431842284037,50372078113535004678$,

$37421019515935943419,28337317234870685051,19675615082715287775$,

$15217135846276911565,11096050540423091781,8696843665586570188$, $6635835606893433938,8696843665586570188,11096050540423091781$, 15217135846276911565,19675615082715287775 , 28337317234870685051 , $37421019515935943419,50372078113535004678\}$

histogram $\{46001200,69816900,107476259,108942109,147098290,152098232$, 206669000, 249209300, 740573600, 816520800, 1353572956, 1513325783, 2748938461, $3004419704,4452940833,4553946490\}$

\section{Input interpretation:}

histogram $\{46001200,69816900,107476259,108942109$, $147098290,152098232,206669000,249209300$, $740573600,816520800,1353572956,1513325783$, $2748938461,3004419704,4452940833,4553946490\}$

\section{Histogram:}

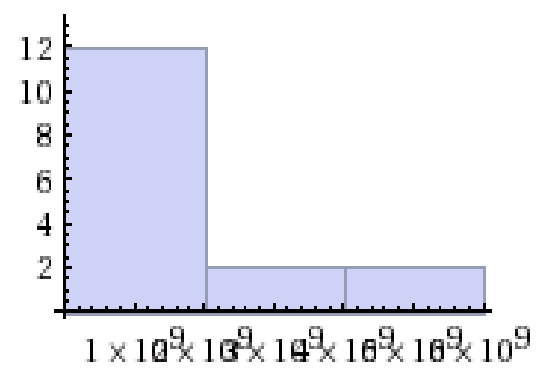

Input:

ToeplitzMatrix [146 001 200, 69816900,107476 259, 108942 109, 147098 290, 152098 232, 206669000,249209 300, $740573600,816520800,1353572956$, $1513325783,2748938461,3004419704,4452940833,4553946490\}]$

\section{Result:}

We shall now give some mathematical evidences that will prove that in the Solar System there really is programmatic and cybernetic algorithm in which it is ,recorded“, in the language of mathematics, how the planets will be built and what will be the quantitative characteristics of the given planetary information. 
Digital Table of the Perihelion and Aphelion

\begin{tabular}{|c|c|c|c|c|c|c|c|}
\hline $\begin{array}{c}\text { Merkur } \\
46001200\end{array}$ & $\begin{array}{l}\text { Merkur } \\
69816900\end{array}$ & $\begin{array}{c}\text { Venus } \\
107476259\end{array}$ & $\begin{array}{c}\text { Venus } \\
108942109\end{array}$ & $\begin{array}{c}\text { Earth } \\
147098290\end{array}$ & $\begin{array}{c}\text { Earth } \\
152098232\end{array}$ & $\begin{array}{c}\text { Mars } \\
206669000\end{array}$ & $\begin{array}{c}\text { Mars } \\
249209300\end{array}$ \\
\hline $\begin{array}{l}\text { Merkur } \\
69816900\end{array}$ & $\begin{array}{c}\text { Merkur } \\
\mathbf{4 6 0 0 1 2 0 0}\end{array}$ & $\begin{array}{l}\text { Merkur } \\
69816900\end{array}$ & $\begin{array}{c}\text { Venus } \\
\mathbf{1 0 7 4 7 6 2 5 9}\end{array}$ & $\begin{array}{c}\text { Venus } \\
108942109\end{array}$ & $\begin{array}{c}\text { Earth } \\
147098290\end{array}$ & $\begin{array}{c}\text { Earth } \\
152098232\end{array}$ & $\begin{array}{c}\text { Mars } \\
206669000\end{array}$ \\
\hline $\begin{array}{c}\text { Venus } \\
107476259\end{array}$ & $\begin{array}{c}\text { Merkur } \\
69816900\end{array}$ & $\begin{array}{c}\text { Merkur } \\
46001200\end{array}$ & $\begin{array}{c}\text { Merkur } \\
69816900\end{array}$ & $\begin{array}{c}\text { Venus } \\
107476259\end{array}$ & $\begin{array}{c}\text { Venus } \\
108942109\end{array}$ & $\begin{array}{c}\text { Earth } \\
147098290\end{array}$ & $\begin{array}{c}\text { Earth } \\
152098232\end{array}$ \\
\hline $\begin{array}{c}\text { Venus } \\
108942109\end{array}$ & $\begin{array}{c}\text { Venus } \\
107476259\end{array}$ & $\begin{array}{c}\text { Merkur } \\
69816900\end{array}$ & $\begin{array}{c}\text { Merkur } \\
46001200\end{array}$ & $\begin{array}{c}\text { Merkur } \\
69816900\end{array}$ & $\begin{array}{c}\text { Venus } \\
107476259\end{array}$ & $\begin{array}{c}\text { Venus } \\
108942109\end{array}$ & $\begin{array}{c}\text { Earth } \\
147098290\end{array}$ \\
\hline $\begin{array}{c}\text { Earth } \\
147098290\end{array}$ & $\begin{array}{c}\text { Venus } \\
108942109\end{array}$ & $\begin{array}{c}\text { Venus } \\
107476259\end{array}$ & $\begin{array}{l}\text { Merkur } \\
69816900\end{array}$ & $\begin{array}{c}\text { Merkur } \\
46001200\end{array}$ & $\begin{array}{l}\text { Merkur } \\
69816900\end{array}$ & $\begin{array}{c}\text { Venus } \\
107476259\end{array}$ & $\begin{array}{c}\text { Venus } \\
108942109\end{array}$ \\
\hline $\begin{array}{c}\text { Earth } \\
152098232\end{array}$ & $\begin{array}{c}\text { Earth } \\
147098290\end{array}$ & $\begin{array}{c}\text { Venus } \\
108942109\end{array}$ & $\begin{array}{c}\text { Venus } \\
107476259\end{array}$ & $\begin{array}{c}\text { Merkur } \\
69816900\end{array}$ & $\begin{array}{c}\text { Merkur } \\
46001200\end{array}$ & $\begin{array}{c}\text { Merkur } \\
69816900\end{array}$ & $\begin{array}{c}\text { Venus } \\
107476259\end{array}$ \\
\hline $\begin{array}{c}\text { Mars } \\
206669000\end{array}$ & $\begin{array}{c}\text { Earth } \\
152098232\end{array}$ & $\begin{array}{c}\text { Earth } \\
147098290\end{array}$ & $\begin{array}{c}\text { Venus } \\
108942109\end{array}$ & $\begin{array}{c}\text { Venus } \\
107476259\end{array}$ & $\begin{array}{l}\text { Merkur } \\
\text { 69816900 }\end{array}$ & $\begin{array}{c}\text { Merkur } \\
\mathbf{4 6 0 0 1 2 0 0}\end{array}$ & $\begin{array}{l}\text { Merkur } \\
69816900\end{array}$ \\
\hline $\begin{array}{c}\text { Mars } \\
249209300\end{array}$ & $\begin{array}{c}\text { Mars } \\
206669000\end{array}$ & $\begin{array}{c}\text { Earth } \\
152098232\end{array}$ & $\begin{array}{c}\text { Earth } \\
147098290\end{array}$ & $\begin{array}{c}\text { Venus } \\
108942109\end{array}$ & $\begin{array}{c}\text { Venus } \\
107476259\end{array}$ & $\begin{array}{c}\text { Merkur } \\
69816900\end{array}$ & $\begin{array}{c}\text { Merkur } \\
46001200\end{array}$ \\
\hline $\begin{array}{c}\text { Jupiter } \\
\mathbf{7 4 0 5 7 3 6 0 0}\end{array}$ & $\begin{array}{c}\text { Mars } \\
249209300\end{array}$ & $\begin{array}{c}\text { Mars } \\
206669000\end{array}$ & $\begin{array}{c}\text { Earth } \\
152098232\end{array}$ & $\begin{array}{c}\text { Earth } \\
147098290\end{array}$ & $\begin{array}{c}\text { Venus } \\
108942109\end{array}$ & $\begin{array}{c}\text { Venus } \\
\mathbf{1 0 7 4 7 6 2 5 9}\end{array}$ & $\begin{array}{l}\text { Merkur } \\
69816900\end{array}$ \\
\hline $\begin{array}{c}\text { Jupiter } \\
\mathbf{8 1 6 5 2 0 8 0 0}\end{array}$ & $\begin{array}{c}\text { Jupiter } \\
\mathbf{7 4 0 5 7 3 6 0 0}\end{array}$ & $\begin{array}{c}\text { Mars } \\
249209300\end{array}$ & $\begin{array}{c}\text { Mars } \\
206669000\end{array}$ & $\begin{array}{c}\text { Earth } \\
152098232\end{array}$ & $\begin{array}{c}\text { Earth } \\
147098290\end{array}$ & $\begin{array}{c}\text { Venus } \\
108942109\end{array}$ & $\begin{array}{c}\text { Venus } \\
107476259\end{array}$ \\
\hline $\begin{array}{c}\text { Saturn } \\
\mathbf{1 3 5 3 5 7 2 9 5 6}\end{array}$ & $\begin{array}{c}\text { Jupiter } \\
\mathbf{8 1 6 5 2 0 8 0 0}\end{array}$ & $\begin{array}{c}\text { Jupiter } \\
\mathbf{7 4 0 5 7 3 6 0 0}\end{array}$ & $\begin{array}{c}\text { Mars } \\
249209300\end{array}$ & $\begin{array}{c}\text { Mars } \\
206669000\end{array}$ & $\begin{array}{c}\text { Earth } \\
152098232\end{array}$ & $\begin{array}{c}\text { Earth } \\
147098290\end{array}$ & $\begin{array}{c}\text { Venus } \\
108942109\end{array}$ \\
\hline $\begin{array}{c}\text { Saturn } \\
\mathbf{1 5 1 3 3 2 5 7 8 3}\end{array}$ & $\begin{array}{c}\text { Saturn } \\
\mathbf{1 3 5 3 5 7 2 9 5 6}\end{array}$ & $\begin{array}{c}\text { Jupiter } \\
\mathbf{8 1 6 5 2 0 8 0 0}\end{array}$ & $\begin{array}{c}\text { Jupiter } \\
\mathbf{7 4 0 5 7 3 6 0 0}\end{array}$ & $\begin{array}{c}\text { Mars } \\
249209300\end{array}$ & $\begin{array}{c}\text { Mars } \\
206669000\end{array}$ & $\begin{array}{c}\text { Earth } \\
152098232\end{array}$ & $\begin{array}{c}\text { Earth } \\
147098290\end{array}$ \\
\hline $\begin{array}{c}\text { Uranus } \\
2748938461\end{array}$ & $\begin{array}{c}\text { Saturn } \\
1513325783\end{array}$ & $\begin{array}{c}\text { Saturn } \\
\mathbf{1 3 5 3 5 7 2 9 5 6}\end{array}$ & $\begin{array}{c}\text { Jupiter } \\
\mathbf{8 1 6 5 2 0 8 0 0}\end{array}$ & $\begin{array}{c}\text { Jupiter } \\
\mathbf{7 4 0 5 7 3 6 0 0}\end{array}$ & $\begin{array}{c}\text { Mars } \\
249209300\end{array}$ & $\begin{array}{c}\text { Mars } \\
206669000\end{array}$ & $\begin{array}{c}\text { Earth } \\
152098232\end{array}$ \\
\hline $\begin{array}{c}\text { Uranus } \\
3004419704\end{array}$ & $\begin{array}{c}\text { Uranus } \\
2748938461\end{array}$ & $\begin{array}{c}\text { Saturn } \\
1513325783\end{array}$ & $\begin{array}{c}\text { Saturn } \\
\mathbf{1 3 5 3 5 7 2 9 5 6}\end{array}$ & $\begin{array}{c}\text { Jupiter } \\
\mathbf{8 1 6 5 2 0 8 0 0}\end{array}$ & $\begin{array}{c}\text { Jupiter } \\
\mathbf{7 4 0 5 7 3 6 0 0}\end{array}$ & $\begin{array}{c}\text { Mars } \\
\mathbf{2 4 9 2 0 9 3 0 0}\end{array}$ & $\begin{array}{c}\text { Mars } \\
206669000\end{array}$ \\
\hline $\begin{array}{c}\text { Neptune } \\
\mathbf{4 4 5 2 9 4 0 8 3 3}\end{array}$ & $\begin{array}{c}\text { Uranus } \\
3004419704\end{array}$ & $\begin{array}{c}\text { Uranus } \\
2748938461\end{array}$ & $\begin{array}{c}\text { Saturn } \\
1513325783\end{array}$ & $\begin{array}{c}\text { Saturn } \\
\mathbf{1 3 5 3 5 7 2 9 5 6}\end{array}$ & $\begin{array}{c}\text { Jupiter } \\
\mathbf{8 1 6 5 2 0 8 0 0}\end{array}$ & $\begin{array}{c}\text { Jupiter } \\
\mathbf{7 4 0 5 7 3 6 0 0}\end{array}$ & $\begin{array}{c}\text { Mars } \\
249209300\end{array}$ \\
\hline $\begin{array}{c}\text { Neptune } \\
\mathbf{4 5 5 3 9 4 6 4 9 0}\end{array}$ & $\begin{array}{c}\text { Neptune } \\
\mathbf{4 4 5 2 9 4 0 8 3 3}\end{array}$ & $\begin{array}{c}\text { Uranus } \\
3004419704\end{array}$ & $\begin{array}{c}\text { Uranus } \\
2748938461\end{array}$ & $\begin{array}{c}\text { Saturn } \\
\mathbf{1 5 1 3 3 2 5 7 8 3}\end{array}$ & $\begin{array}{c}\text { Saturn } \\
\mathbf{1 3 5 3 5 7 2 9 5 6}\end{array}$ & $\begin{array}{c}\text { Jupiter } \\
\mathbf{8 1 6 5 2 0 8 0 0}\end{array}$ & $\begin{array}{c}\text { Jupiter } \\
\mathbf{7 4 0 5 7 3 6 0 0}\end{array}$ \\
\hline 20271549917 & 15787420327 & 11441955753 & 8546478158 & 5944637987 & 4583410436 & 3436506480 & 2869194980 \\
\hline
\end{tabular}

\begin{tabular}{|c|c|c|c|c|c|c|c|}
\hline $\begin{array}{c}\text { Jupiter } \\
\mathbf{7 4 0 5 7 3 6 0 0}\end{array}$ & $\begin{array}{c}\text { Jupiter } \\
\mathbf{8 1 6 5 2 0 8 0 0}\end{array}$ & $\begin{array}{c}\text { Saturn } \\
\mathbf{1 3 5 3 5 7 2 9 5 6}\end{array}$ & $\begin{array}{c}\text { Saturn } \\
\mathbf{1 5 1 3 3 2 5 7 8 3}\end{array}$ & $\begin{array}{c}\text { Uranus } \\
2748938461\end{array}$ & $\begin{array}{c}\text { Uranus } \\
\mathbf{3 0 0 4 4 1 9 7 0 4}\end{array}$ & $\begin{array}{l}\text { Neptune } \\
\mathbf{4 4 5 2 9 4 0 8 3 3}\end{array}$ & $\begin{array}{l}\text { Neptune } \\
\mathbf{4 5 5 3 9 4 6 4 9 0}\end{array}$ \\
\hline $\begin{array}{c}\text { Mars } \\
249209300\end{array}$ & $\begin{array}{c}\text { Jupiter } \\
\mathbf{7 4 0 5 7 3 6 0 0}\end{array}$ & $\begin{array}{c}\text { Jupiter } \\
\mathbf{8 1 6 5 2 0 8 0 0}\end{array}$ & $\begin{array}{c}\text { Satur } \\
1353572956\end{array}$ & $\begin{array}{c}\text { Saturn } \\
1513325783\end{array}$ & $\begin{array}{c}\text { Uranus } \\
2748938461\end{array}$ & $\begin{array}{c}\text { Uranus } \\
\mathbf{3 0 0 4 4 1 9 7 0 4}\end{array}$ & $\begin{array}{c}\text { Neptune } \\
\mathbf{4 4 5 2 9 4 0 8 3 3}\end{array}$ \\
\hline $\begin{array}{c}\text { Mar } \\
206669000\end{array}$ & $\begin{array}{c}\text { Mars } \\
249209300\end{array}$ & $\begin{array}{c}\text { Jupiter } \\
740573600\end{array}$ & $\begin{array}{c}\text { Jupiter } \\
\mathbf{8 1 6 5 2 0 8 0 0}\end{array}$ & $\begin{array}{c}\text { Saturn } \\
\mathbf{1 3 5 3 5 7 2 9 5 6}\end{array}$ & $\begin{array}{c}\text { Saturn } \\
1513325783\end{array}$ & $\begin{array}{c}\text { Uranus } \\
2748938461\end{array}$ & $\begin{array}{c}\text { Uranus } \\
\mathbf{3 0 0 4 4 1 9 7 0 4}\end{array}$ \\
\hline $\begin{array}{c}\text { Earth } \\
152098232\end{array}$ & $\begin{array}{c}\text { Mars } \\
206669000\end{array}$ & $\begin{array}{c}\text { Mars } \\
249209300\end{array}$ & $\begin{array}{c}\text { Jupiter } \\
\mathbf{7 4 0 5 7 3 6 0 0}\end{array}$ & $\begin{array}{c}\text { Jupiter } \\
\mathbf{8 1 6 5 2 0 8 0 0}\end{array}$ & $\begin{array}{c}\text { Saturn } \\
\mathbf{1 3 5 3 5 7 2 9 5 6}\end{array}$ & $\begin{array}{c}\text { Saturn } \\
\mathbf{1 5 1 3 3 2 5 7 8 3}\end{array}$ & $\begin{array}{c}\text { Uranus } \\
2748938461\end{array}$ \\
\hline $\begin{array}{c}\text { Earth } \\
147098290\end{array}$ & $\begin{array}{c}\text { Earth } \\
152098232\end{array}$ & $\begin{array}{c}\text { Mars } \\
206669000\end{array}$ & $\begin{array}{c}\text { Mars } \\
249209300\end{array}$ & $\begin{array}{c}\text { Jupiter } \\
740573600\end{array}$ & $\begin{array}{c}\text { Jupiter } \\
\mathbf{8 1 6 5 2 0 8 0 0}\end{array}$ & $\begin{array}{c}\text { Saturn } \\
\mathbf{1 3 5 3 5 7 2 9 5 6}\end{array}$ & $\begin{array}{c}\text { Saturn } \\
\mathbf{1 5 1 3 3 2 5 7 8 3}\end{array}$ \\
\hline $\begin{array}{c}\text { Mars } \\
108942109\end{array}$ & $\begin{array}{c}\text { Earth } \\
147098290\end{array}$ & $\begin{array}{c}\text { Earth } \\
152098232\end{array}$ & $\begin{array}{c}\text { Mars } \\
206669000\end{array}$ & $\begin{array}{c}\text { Mars } \\
249209300\end{array}$ & $\begin{array}{c}\text { Jupiter } \\
\mathbf{7 4 0 5 7 3 6 0 0}\end{array}$ & $\begin{array}{c}\text { Jupiter } \\
\mathbf{8 1 6 5 2 0 8 0 0 0}\end{array}$ & $\begin{array}{c}\text { Saturn } \\
1353572956\end{array}$ \\
\hline $\begin{array}{c}\text { Venus } \\
107476259\end{array}$ & $\begin{array}{c}\text { Mars } \\
108942109\end{array}$ & $\begin{array}{c}\text { Earth } \\
147098290\end{array}$ & $\begin{array}{c}\text { Earth } \\
152098232\end{array}$ & $\begin{array}{c}\text { Mars } \\
206669000\end{array}$ & $\begin{array}{c}\text { Mars } \\
249209300\end{array}$ & $\begin{array}{c}\text { Jupiter } \\
\mathbf{7 4 0 5 7 3 6 0 0}\end{array}$ & $\begin{array}{c}\text { Jupiter } \\
\mathbf{8 1 6 5 2 0 8 0 0}\end{array}$ \\
\hline $\begin{array}{c}\text { Merkur } \\
69816900\end{array}$ & $\begin{array}{c}\text { Venus } \\
107476259\end{array}$ & $\begin{array}{c}\text { Mars } \\
108942109\end{array}$ & $\begin{array}{c}\text { Earth } \\
147098290\end{array}$ & $\begin{array}{c}\text { Earth } \\
152098232\end{array}$ & $\begin{array}{c}\text { Mars } \\
206669000\end{array}$ & $\begin{array}{c}\text { Mars } \\
249209300\end{array}$ & $\begin{array}{c}\text { Jupiter } \\
\mathbf{7 4 0 5 7 3 6 0 0}\end{array}$ \\
\hline $\begin{array}{c}\text { Merkur } \\
\mathbf{4 6 0 0 1 2 0 0}\end{array}$ & $\begin{array}{c}\text { Merkur } \\
69816900\end{array}$ & $\begin{array}{c}\text { Venus } \\
107476259\end{array}$ & $\begin{array}{c}\text { Mars } \\
108942109\end{array}$ & $\begin{array}{c}\text { Earth } \\
147098290\end{array}$ & $\begin{array}{c}\text { Earth } \\
152098232\end{array}$ & $\begin{array}{c}\text { Mars } \\
206669000\end{array}$ & $\begin{array}{c}\text { Mars } \\
249209300\end{array}$ \\
\hline $\begin{array}{c}\text { Merkur } \\
69816900\end{array}$ & $\begin{array}{c}\text { Merkur } \\
46001200\end{array}$ & $\begin{array}{c}\text { Merkur } \\
69816900\end{array}$ & $\begin{array}{c}\text { Venus } \\
107476259\end{array}$ & $\begin{array}{c}\text { Mars } \\
108942109\end{array}$ & $\begin{array}{c}\text { Earth } \\
147098290\end{array}$ & $\begin{array}{c}\text { Earth } \\
152098232\end{array}$ & $\begin{array}{c}\text { Mars } \\
206669000\end{array}$ \\
\hline $\begin{array}{c}\text { Venus } \\
107476259\end{array}$ & $\begin{array}{c}\text { Merkur } \\
69816900\end{array}$ & $\begin{array}{r}\text { Merkur } \\
46001200\end{array}$ & $\begin{array}{c}\text { Merkur } \\
69816900\end{array}$ & $\begin{array}{c}\text { Venus } \\
107476259\end{array}$ & $\begin{array}{c}\text { Mars } \\
108942109\end{array}$ & $\begin{array}{c}\text { Earth } \\
147098290\end{array}$ & $\begin{array}{c}\text { Earth } \\
152098232\end{array}$ \\
\hline $\begin{array}{c}\text { Mars } \\
108942109\end{array}$ & $\begin{array}{c}\text { Venus } \\
107476259\end{array}$ & $\begin{array}{c}\text { Merkur } \\
69816900\end{array}$ & $\begin{array}{c}\text { Merkur } \\
\mathbf{4 6 0 0 1 2 0 0}\end{array}$ & $\begin{array}{c}\text { Merkur } \\
69816900\end{array}$ & $\begin{array}{c}\text { Venus } \\
107476259\end{array}$ & $\begin{array}{c}\text { Mars } \\
108942109\end{array}$ & $\begin{array}{c}\text { Earth } \\
147098290\end{array}$ \\
\hline
\end{tabular}




\begin{tabular}{|c|c|c|c|c|c|c|c|}
\hline $\begin{array}{c}\text { Earth } \\
147098290\end{array}$ & $\begin{array}{c}\text { Mars } \\
108942109\end{array}$ & $\begin{array}{c}\text { Venus } \\
107476259\end{array}$ & $\begin{array}{c}\text { Merkur } \\
69816900\end{array}$ & $\begin{array}{l}\text { Merkur } \\
\mathbf{4 6 0 0 1 2 0 0}\end{array}$ & $\begin{array}{c}\text { Merkur } \\
69816900\end{array}$ & $\begin{array}{c}\text { Venus } \\
107476259\end{array}$ & $\begin{array}{c}\text { Mars } \\
108942109\end{array}$ \\
\hline $\begin{array}{c}\text { Earth } \\
152098232\end{array}$ & $\begin{array}{c}\text { Earth } \\
147098290\end{array}$ & $\begin{array}{c}\text { Mars } \\
108942109\end{array}$ & $\begin{array}{c}\text { Venus } \\
\mathbf{1 0 7 4 7 6 2 5 9}\end{array}$ & $\begin{array}{l}\text { Merkur } \\
69816900\end{array}$ & $\begin{array}{c}\text { Merkur } \\
\mathbf{4 6 0 0 1 2 0 0}\end{array}$ & $\begin{array}{l}\text { Merkur } \\
69816900\end{array}$ & $\begin{array}{c}\text { Venus } \\
107476259\end{array}$ \\
\hline $\begin{array}{c}\text { Mars } \\
206669000\end{array}$ & $\begin{array}{c}\text { Earth } \\
152098232\end{array}$ & $\begin{array}{c}\text { Earth } \\
147098290\end{array}$ & $\begin{array}{c}\text { Mars } \\
108942109\end{array}$ & $\begin{array}{c}\text { Venus } \\
107476259\end{array}$ & $\begin{array}{l}\text { Merkur } \\
69816900\end{array}$ & $\begin{array}{c}\text { Merkur } \\
46001200\end{array}$ & $\begin{array}{l}\text { Merkur } \\
69816900\end{array}$ \\
\hline $\begin{array}{c}\text { Mars } \\
\mathbf{2 4 9 2 0 9 3 0 0} \\
\end{array}$ & $\begin{array}{c}\text { Mars } \\
206669000\end{array}$ & $\begin{array}{c}\text { Earth } \\
152098232\end{array}$ & $\begin{array}{c}\text { Earth } \\
147098290 \\
\end{array}$ & $\begin{array}{c}\text { Mars } \\
108942109\end{array}$ & $\begin{array}{c}\text { Venus } \\
107476259 \\
\end{array}$ & $\begin{array}{l}\text { Merkur } \\
69816900 \\
\end{array}$ & $\begin{array}{c}\text { Merkur } \\
\mathbf{4 6 0 0 1 2 0 0} \\
\end{array}$ \\
\hline 2869194980 & 3436506480 & 4583410436 & 5944637987 & 8546478158 & 11441955753 & 15787420327 & 20271549917 \\
\hline
\end{tabular}

Within the digital pictures in astronomy, the physical and chemical parameters are in a strict compliance with programmatic, cybernetic and information principles. The digital language of astronomy has a countless number of codes and analogue codes, as well as other information content. These pictures enable us to realize the very essence of functioning of astronomical phenomenons (Orbital characteristics, physical characteristics, etc.). There are some examples:

\section{Digital correlations of the Perihelion and Aphelion:}

\begin{tabular}{|c|c|c|c|c|c|c|c|}
\hline $\begin{array}{c}\text { Earth } \\
147098290\end{array}$ & $\begin{array}{c}\text { Earth } \\
152098232\end{array}$ & $\begin{array}{c}\text { Mars } \\
206669000\end{array}$ & $\begin{array}{c}\text { Mars } \\
249209300\end{array}$ & $\begin{array}{c}\text { Jupiter } \\
\mathbf{7 4 0 5 7 3 6 0 0}\end{array}$ & $\begin{array}{c}\text { Jupiter } \\
\mathbf{8 1 6 5 2 0 8 0 0}\end{array}$ & $\begin{array}{c}\text { Saturn } \\
1353572956\end{array}$ & $\begin{array}{c}\text { Saturn } \\
1513325783\end{array}$ \\
\hline $\begin{array}{c}\text { Venus } \\
108942109\end{array}$ & $\begin{array}{c}\text { Earth } \\
147098290\end{array}$ & $\begin{array}{c}\text { Earth } \\
152098232\end{array}$ & $\begin{array}{c}\text { Mars } \\
\mathbf{2 0 6 6 6 9 0 0 0}\end{array}$ & $\begin{array}{c}\text { Mars } \\
\mathbf{2 4 9 2 0 9 3 0 0}\end{array}$ & $\begin{array}{c}\text { Jupiter } \\
\mathbf{7 4 0 5 7 3 6 0 0}\end{array}$ & $\begin{array}{c}\text { Jupiter } \\
\mathbf{8 1 6 5 2 0 8 0 0}\end{array}$ & $\begin{array}{c}\text { Satur } \\
\mathbf{1 3 5 3 5 7 2 9 5 6}\end{array}$ \\
\hline $\begin{array}{c}\text { Venus } \\
107476259\end{array}$ & $\begin{array}{c}\text { Venus } \\
108942109\end{array}$ & $\begin{array}{c}\text { Earth } \\
147098290\end{array}$ & $\begin{array}{c}\text { Earth } \\
152098232\end{array}$ & $\begin{array}{c}\text { Mar } \\
206669000\end{array}$ & $\begin{array}{c}\text { Mars } \\
249209300\end{array}$ & $\begin{array}{c}\text { Jupiter } \\
\mathbf{7 4 0 5 7 3 6 0 0}\end{array}$ & $\begin{array}{c}\text { Jupiter } \\
\mathbf{8 1 6 5 2 0 8 0 0}\end{array}$ \\
\hline $\begin{array}{c}\text { Merkur } \\
69816900\end{array}$ & $\begin{array}{c}\text { Venus } \\
107476259\end{array}$ & $\begin{array}{c}\text { Venus } \\
108942109\end{array}$ & $\begin{array}{c}\text { Earth } \\
147098290\end{array}$ & $\begin{array}{c}\text { Earth } \\
152098232\end{array}$ & $\begin{array}{c}\text { Mars } \\
\mathbf{2 0 6 6 6 9 0 0 0}\end{array}$ & $\begin{array}{c}\text { Mars } \\
\mathbf{2 4 9 2 0 9 3 0 0}\end{array}$ & $\begin{array}{c}\text { Jupiter } \\
740573600\end{array}$ \\
\hline $\begin{array}{c}\text { Merkur } \\
46001200\end{array}$ & $\begin{array}{l}\text { Merkur } \\
69816900\end{array}$ & $\begin{array}{c}\text { Venus } \\
107476259\end{array}$ & $\begin{array}{c}\text { Venus } \\
108942109\end{array}$ & $\begin{array}{c}\text { Earth } \\
147098290\end{array}$ & $\begin{array}{c}\text { Earth } \\
152098232\end{array}$ & $\begin{array}{c}\text { Mars } \\
206669000\end{array}$ & $\begin{array}{c}\text { Mars } \\
249209300\end{array}$ \\
\hline $\begin{array}{c}\text { Merkur } \\
69816900\end{array}$ & $\begin{array}{l}\text { Merkur } \\
\mathbf{4 6 0 0 1 2 0 0}\end{array}$ & $\begin{array}{l}\text { Merkur } \\
69816900\end{array}$ & $\begin{array}{c}\text { Venus } \\
107476259\end{array}$ & $\begin{array}{c}\text { Mars } \\
\mathbf{1 0 8 9 4 2 1 0 9}\end{array}$ & $\begin{array}{c}\text { Earth } \\
147098290\end{array}$ & $\begin{array}{c}\text { Earth } \\
152098232\end{array}$ & $\begin{array}{c}\text { Mars } \\
\mathbf{2 0 6 6 6 9 0 0 0}\end{array}$ \\
\hline $\begin{array}{c}\text { Venus } \\
\mathbf{1 0 7 4 7 6 2 5 9}\end{array}$ & $\begin{array}{l}\text { Merkur } \\
69816900\end{array}$ & $\begin{array}{l}\text { Merkur } \\
46001200\end{array}$ & $\begin{array}{c}\text { Merkur } \\
69816900\end{array}$ & $\begin{array}{c}\text { Venus } \\
107476259\end{array}$ & $\begin{array}{c}\text { Mars } \\
\mathbf{1 0 8 9 4 2 1 0 9}\end{array}$ & $\begin{array}{c}\text { Earth } \\
147098290\end{array}$ & $\begin{array}{c}\text { Earth } \\
152098232\end{array}$ \\
\hline $\begin{array}{c}\text { Venus } \\
108942109\end{array}$ & $\begin{array}{c}\text { Venus } \\
107476259\end{array}$ & $\begin{array}{l}\text { Merkur } \\
69816900\end{array}$ & $\begin{array}{l}\text { Merkur } \\
46001200\end{array}$ & $\begin{array}{l}\text { Merkur } \\
69816900\end{array}$ & $\begin{array}{c}\text { Venus } \\
\mathbf{1 0 7 4 7 6 2 5 9}\end{array}$ & $\begin{array}{c}\text { Mars } \\
\mathbf{1 0 8 9 4 2 1 0 9}\end{array}$ & $\begin{array}{c}\text { Earth } \\
147098290\end{array}$ \\
\hline $\begin{array}{c}\text { Earth } \\
147098290\end{array}$ & $\begin{array}{c}\text { Venus } \\
108942109\end{array}$ & $\begin{array}{c}\text { Venus } \\
107476259\end{array}$ & $\begin{array}{l}\text { Merkur } \\
69816900\end{array}$ & $\begin{array}{l}\text { Merkur } \\
46001200\end{array}$ & $\begin{array}{l}\text { Merkur } \\
69816900\end{array}$ & $\begin{array}{c}\text { Venus } \\
107476259\end{array}$ & $\begin{array}{c}\text { Mars } \\
108942109\end{array}$ \\
\hline $\begin{array}{c}\text { Earth } \\
152098232\end{array}$ & $\begin{array}{c}\text { Earth } \\
147098290\end{array}$ & $\begin{array}{c}\text { Venus } \\
108942109\end{array}$ & $\begin{array}{c}\text { Venus } \\
107476259\end{array}$ & $\begin{array}{l}\text { Merkur } \\
69816900\end{array}$ & $\begin{array}{l}\text { Merkur } \\
46001200\end{array}$ & $\begin{array}{c}\text { Merkur } \\
69816900\end{array}$ & $\begin{array}{c}\text { Venus } \\
107476259\end{array}$ \\
\hline $\begin{array}{c}\text { Mars } \\
\mathbf{2 0 6 6 6 9 0 0 0}\end{array}$ & $\begin{array}{c}\text { Earth } \\
152098232\end{array}$ & $\begin{array}{c}\text { Earth } \\
147098290\end{array}$ & $\begin{array}{c}\text { Venus } \\
108942109\end{array}$ & $\begin{array}{c}\text { Venus } \\
107476259\end{array}$ & $\begin{array}{l}\text { Merkur } \\
69816900\end{array}$ & $\begin{array}{l}\text { Merkur } \\
46001200\end{array}$ & $\begin{array}{l}\text { Merkur } \\
69816900\end{array}$ \\
\hline $\begin{array}{c}\text { Mars } \\
\mathbf{2 4 9 2 0 9 3 0 0}\end{array}$ & $\begin{array}{c}\text { Mars } \\
\mathbf{2 0 6 6 6 9 0 0 0}\end{array}$ & $\begin{array}{c}\text { Earth } \\
152098232\end{array}$ & $\begin{array}{c}\text { Earth } \\
147098290\end{array}$ & $\begin{array}{c}\text { Mars } \\
108942109\end{array}$ & $\begin{array}{c}\text { Venus } \\
107476259\end{array}$ & $\begin{array}{l}\text { Merkur } \\
69816900\end{array}$ & $\begin{array}{c}\text { Merkur } \\
46001200\end{array}$ \\
\hline $\begin{array}{c}\text { Jupiter } \\
740573600\end{array}$ & $\begin{array}{c}\text { Mars } \\
\mathbf{2 4 9 2 0 9 3 0 0}\end{array}$ & $\begin{array}{c}\text { Mars } \\
\mathbf{2 0 6 6 6 9 0 0 0}\end{array}$ & $\begin{array}{c}\text { Earth } \\
152098232\end{array}$ & $\begin{array}{c}\text { Earth } \\
147098290\end{array}$ & $\begin{array}{c}\text { Mars } \\
\mathbf{1 0 8 9 4 2 1 0 9}\end{array}$ & $\begin{array}{c}\text { Venus } \\
\mathbf{1 0 7 4 7 6 2 5 9}\end{array}$ & $\begin{array}{c}\text { Merkur } \\
69816900\end{array}$ \\
\hline $\begin{array}{c}\text { Jupiter } \\
\mathbf{8 1 6 5 2 0 8 0 0}\end{array}$ & $\begin{array}{c}\text { Jupiter } \\
\mathbf{7 4 0 5 7 3 6 0 0}\end{array}$ & $\begin{array}{c}\text { Mars } \\
\mathbf{2 4 9 2 0 9 3 0 0}\end{array}$ & $\begin{array}{c}\text { Mars } \\
\mathbf{2 0 6 6 6 9 0 0 0}\end{array}$ & $\begin{array}{c}\text { Earth } \\
152098232\end{array}$ & $\begin{array}{c}\text { Earth } \\
147098290\end{array}$ & $\begin{array}{c}\text { Mars } \\
108942109\end{array}$ & $\begin{array}{c}\text { Venus } \\
107476259\end{array}$ \\
\hline Saturn & Jupiter & Jupiter & Mars & Mars & Earth & Earth & Mars \\
\hline
\end{tabular}




\begin{tabular}{c|c|c|c|c|c|c|c|}
1353572956 & $\mathbf{8 1 6 5 2 0 8 0 0}$ & 740573600 & 249209300 & 206669000 & 152098232 & 147098290 & 108942109 \\
\hline Saturn & Saturn & Jupiter & Jupiter & Mars & Mars & Earth & Earth \\
1513325783 & 1353572956 & 816520800 & 740573600 & 249209300 & 206669000 & 152098232 & 147098290 \\
\hline 5944637987 & 4583410436 & 3436506480 & 2869194980 & 2869194980 & 3436506480 & 4583410436 & 5944637987
\end{tabular}

A these digital Table of order $n$ is an arrangement of $n^{2}$ numbers, usually distinct integers, in a square, such that the $n$ numbers in all rows, all columns, and both diagonals sum to the same constant. A digital square contains the integers from 1 to $n^{2}$. The term "digital square" is also sometimes used to refer to any of various types of word square.

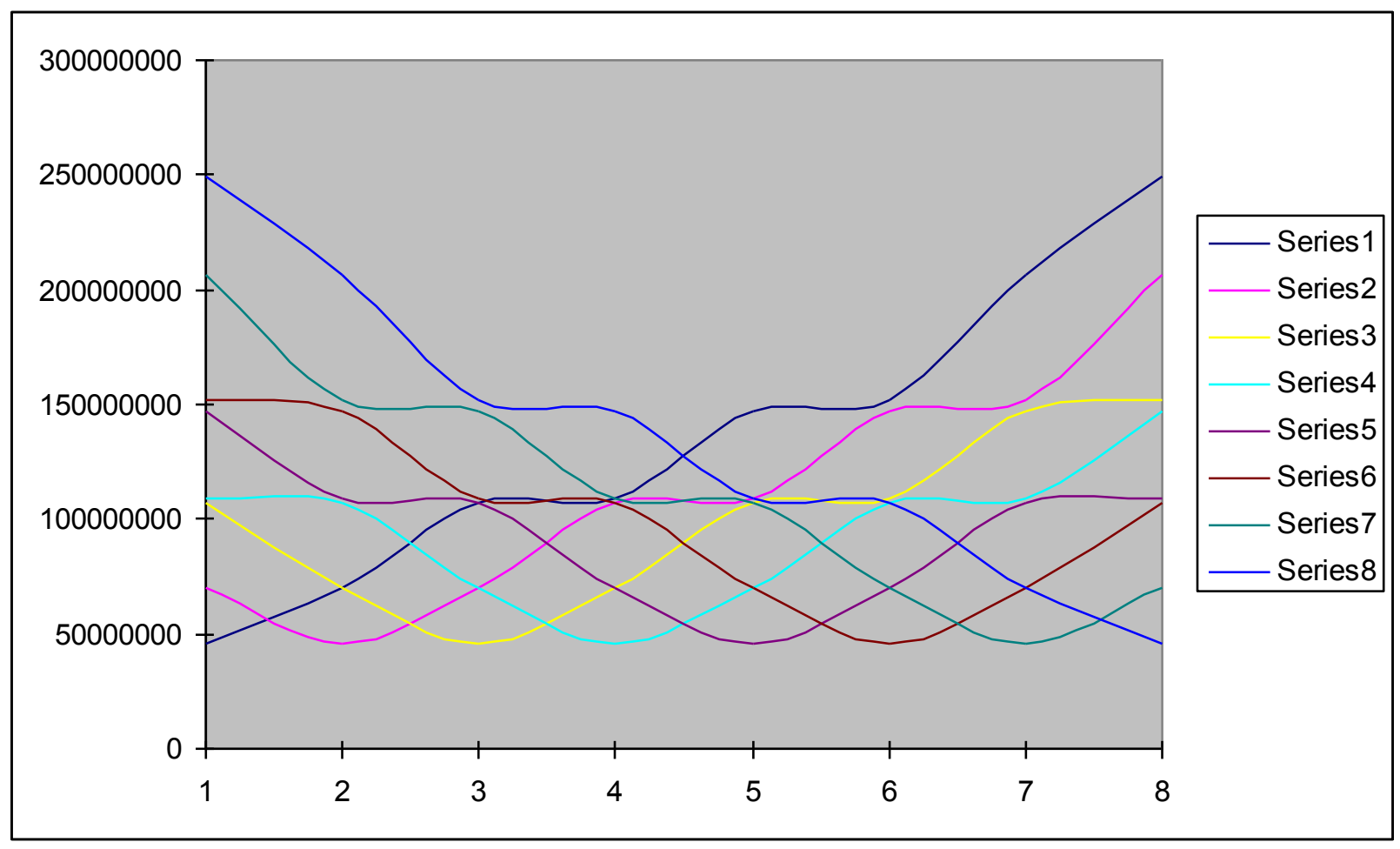

Fig. 1. Schematic representation of the Digital Table of Solar System.

Establishing of numeric values of the Perihelion and Aphelion to be done through use of strictly determined criterion from the theory of systems and also from cybernetics whish, in this example, is the Perihelion and Aphelion. That is only one dimension of the digital image of Solar System. There are many other dimensions as well as digital images. Each of these dimensions and images has its corresponding progression. With some dimensions, one has to use some other parameters from the theory of systems and cybernetics (frequency, standard deviation, various codes and analogue codes, analogue values, primary and secondary values, odd-even relation, and many others), and not progression.

Regardless of the fact whether there is a typical correlation between parameters or not, their effect in the process of formation of the Solar System can be followed through use of adequate methodology. Examples: 
The middle part of the digital table Solar system:

\begin{tabular}{|c|c|c|c|c|c|c|c|}
\hline $\begin{array}{l}\text { Merkur } \\
46001200\end{array}$ & $\begin{array}{l}\text { Merkur } \\
69816900\end{array}$ & $\begin{array}{c}\text { Venus } \\
107476259\end{array}$ & $\begin{array}{c}\text { Venus } \\
108942109\end{array}$ & $\begin{array}{c}\text { Earth } \\
147098290\end{array}$ & $\begin{array}{c}\text { Earth } \\
152098232\end{array}$ & $\begin{array}{c}\text { Mars } \\
206669000\end{array}$ & $\begin{array}{c}\text { Mars } \\
249209300\end{array}$ \\
\hline $\begin{array}{l}\text { Merkur } \\
69816900\end{array}$ & $\begin{array}{l}\text { Merkur } \\
46001200\end{array}$ & $\begin{array}{l}\text { Merkur } \\
69816900\end{array}$ & $\begin{array}{c}\text { Venus } \\
\mathbf{1 0 7 4 7 6 2 5 9}\end{array}$ & $\begin{array}{c}\text { Mars } \\
108942109\end{array}$ & $\begin{array}{c}\text { Earth } \\
147098290\end{array}$ & $\begin{array}{c}\text { Earth } \\
152098232\end{array}$ & $\begin{array}{c}\text { Mars } \\
\mathbf{2 0 6 6 6 9 0 0 0}\end{array}$ \\
\hline $\begin{array}{c}\text { Venus } \\
107476259\end{array}$ & $\begin{array}{l}\text { Merkur } \\
69816900\end{array}$ & $\begin{array}{l}\text { Merkur } \\
46001200\end{array}$ & $\begin{array}{l}\text { Merkur } \\
69816900\end{array}$ & $\begin{array}{c}\text { Venus } \\
107476259\end{array}$ & $\begin{array}{c}\text { Mars } \\
108942109\end{array}$ & $\begin{array}{c}\text { Earth } \\
147098290\end{array}$ & $\begin{array}{c}\text { Earth } \\
152098232\end{array}$ \\
\hline $\begin{array}{c}\text { Venus } \\
108942109\end{array}$ & $\begin{array}{c}\text { Venus } \\
107476259\end{array}$ & $\begin{array}{l}\text { Merkur } \\
69816900\end{array}$ & $\begin{array}{l}\text { Merkur } \\
46001200\end{array}$ & $\begin{array}{l}\text { Merkur } \\
69816900\end{array}$ & $\begin{array}{c}\text { Venus } \\
107476259\end{array}$ & $\begin{array}{c}\text { Mars } \\
108942109\end{array}$ & $\begin{array}{c}\text { Earth } \\
147098290\end{array}$ \\
\hline $\begin{array}{c}\text { Earth } \\
147098290\end{array}$ & $\begin{array}{c}\text { Venus } \\
108942109\end{array}$ & $\begin{array}{c}\text { Venus } \\
107476259\end{array}$ & $\begin{array}{l}\text { Merkur } \\
69816900\end{array}$ & $\begin{array}{l}\text { Merkur } \\
46001200\end{array}$ & $\begin{array}{l}\text { Merkur } \\
69816900\end{array}$ & $\begin{array}{c}\text { Venus } \\
107476259\end{array}$ & $\begin{array}{c}\text { Mars } \\
\mathbf{1 0 8 9 4 2 1 0 9}\end{array}$ \\
\hline $\begin{array}{c}\text { Earth } \\
152098232\end{array}$ & $\begin{array}{c}\text { Earth } \\
147098290\end{array}$ & $\begin{array}{c}\text { Venus } \\
108942109\end{array}$ & $\begin{array}{c}\text { Venus } \\
\mathbf{1 0 7 4 7 6 2 5 9}\end{array}$ & $\begin{array}{l}\text { Merkur } \\
69816900\end{array}$ & $\begin{array}{l}\text { Merkur } \\
46001200\end{array}$ & $\begin{array}{l}\text { Merkur } \\
69816900\end{array}$ & $\begin{array}{c}\text { Venus } \\
107476259\end{array}$ \\
\hline $\begin{array}{c}\text { Mars } \\
\mathbf{2 0 6 6 6 9 0 0 0}\end{array}$ & $\begin{array}{c}\text { Earth } \\
152098232\end{array}$ & $\begin{array}{c}\text { Earth } \\
147098290\end{array}$ & $\begin{array}{c}\text { Venus } \\
108942109\end{array}$ & $\begin{array}{c}\text { Venus } \\
107476259\end{array}$ & $\begin{array}{l}\text { Merkur } \\
69816900\end{array}$ & $\begin{array}{l}\text { Merkur } \\
\mathbf{4 6 0 0 1 2 0 0}\end{array}$ & $\begin{array}{l}\text { Merkur } \\
69816900\end{array}$ \\
\hline $\begin{array}{c}\text { Mars } \\
\mathbf{2 4 9 2 0 9 3 0 0}\end{array}$ & $\begin{array}{c}\text { Mars } \\
\mathbf{2 0 6 6 6 9 0 0 0}\end{array}$ & $\begin{array}{c}\text { Earth } \\
152098232\end{array}$ & $\begin{array}{c}\text { Earth } \\
147098290\end{array}$ & $\begin{array}{c}\text { Mars } \\
108942109\end{array}$ & $\begin{array}{c}\text { Venus } \\
107476259\end{array}$ & $\begin{array}{l}\text { Merkur } \\
69816900\end{array}$ & $\begin{array}{c}\text { Merkur } \\
46001200\end{array}$ \\
\hline 10873111290 & 907918890 & 808726149 & 765570026 & 765570026 & 808726149 & 907918890 & 10873111290 \\
\hline
\end{tabular}

\begin{tabular}{|c|c|c|c|c|c|c|c|}
\hline 46001200 & 69816900 & 107476259 & 108942109 & 147098290 & 152098232 & 206669000 & 249209300 \\
\hline 69816900 & 46001200 & 69816900 & 107476259 & 108942109 & 147098290 & 152098232 & 206669000 \\
\hline 107476259 & 69816900 & 46001200 & 69816900 & 107476259 & 108942109 & 147098290 & 152098232 \\
\hline 108942109 & 107476259 & 69816900 & 46001200 & 69816900 & 107476259 & 108942109 & 147098290 \\
\hline 147098290 & 108942109 & 107476259 & 69816900 & 46001200 & 69816900 & 107476259 & 108942109 \\
\hline 152098232 & 147098290 & 108942109 & 107476259 & 69816900 & 46001200 & 69816900 & 107476259 \\
\hline 206669000 & 152098232 & 147098290 & 108942109 & 107476259 & 69816900 & 46001200 & 69816900 \\
\hline 249209300 & 206669000 & 152098232 & 147098290 & 108942109 & 107476259 & 69816900 & 46001200 \\
\hline 10873111290 & 907918890 & 808726149 & 765570026 & 765570026 & 808726149 & 907918890 & 10873111290 \\
\hline
\end{tabular}

etc.

Making a sequence of all phenomena in digital Solar System is conducted according to the exact mathematical laws (for such descriptions we can use theory of systems and cybernetics.

\section{There are some aexamples:}

\section{Dimensions:}

16 (rows) $\times 16$ (columns) 


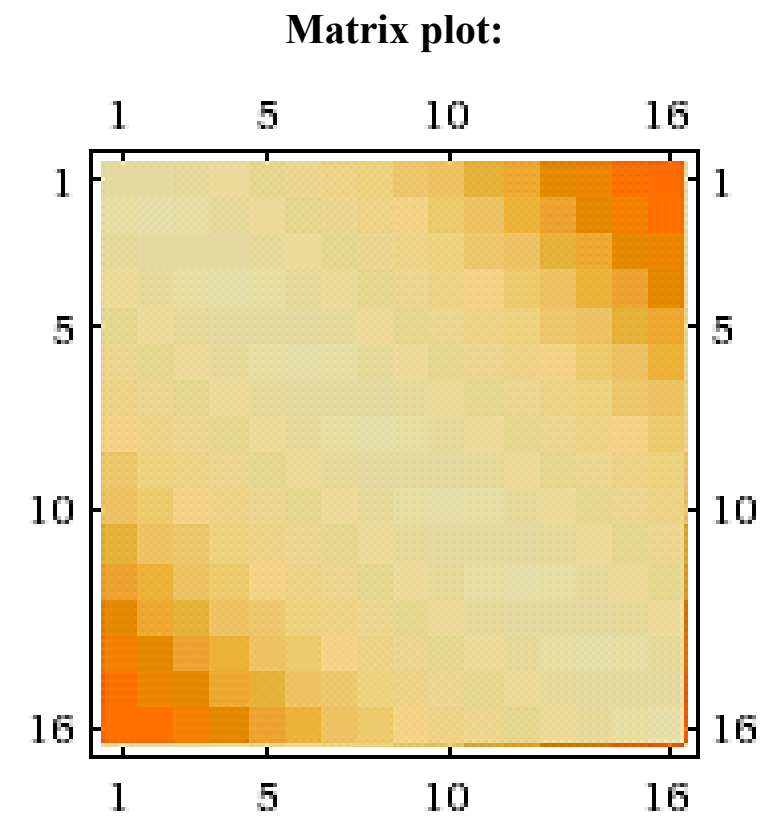

Properties:

symmetric

toeplitz

\section{Determinant:}

$1164852029313230771321512058359970923039267648388578875554946325^{\circ}$ : 013660473893811062257961606057897145785851037437041290850391037 : 568216078373

Trace:

736019200

\section{Condition number:}

693.258

The results of our research show that the processes of sequencing the Solar system are conditioned and arranged not only with orbital characteristics of planets, but also with program, cybernetic and informational lawfulness too. Translation of the cosmic language of these table into a digital language may be very useful for developing new methods of predicting of phenomenon in astronomy. 


\section{Characteristic polynomial:}

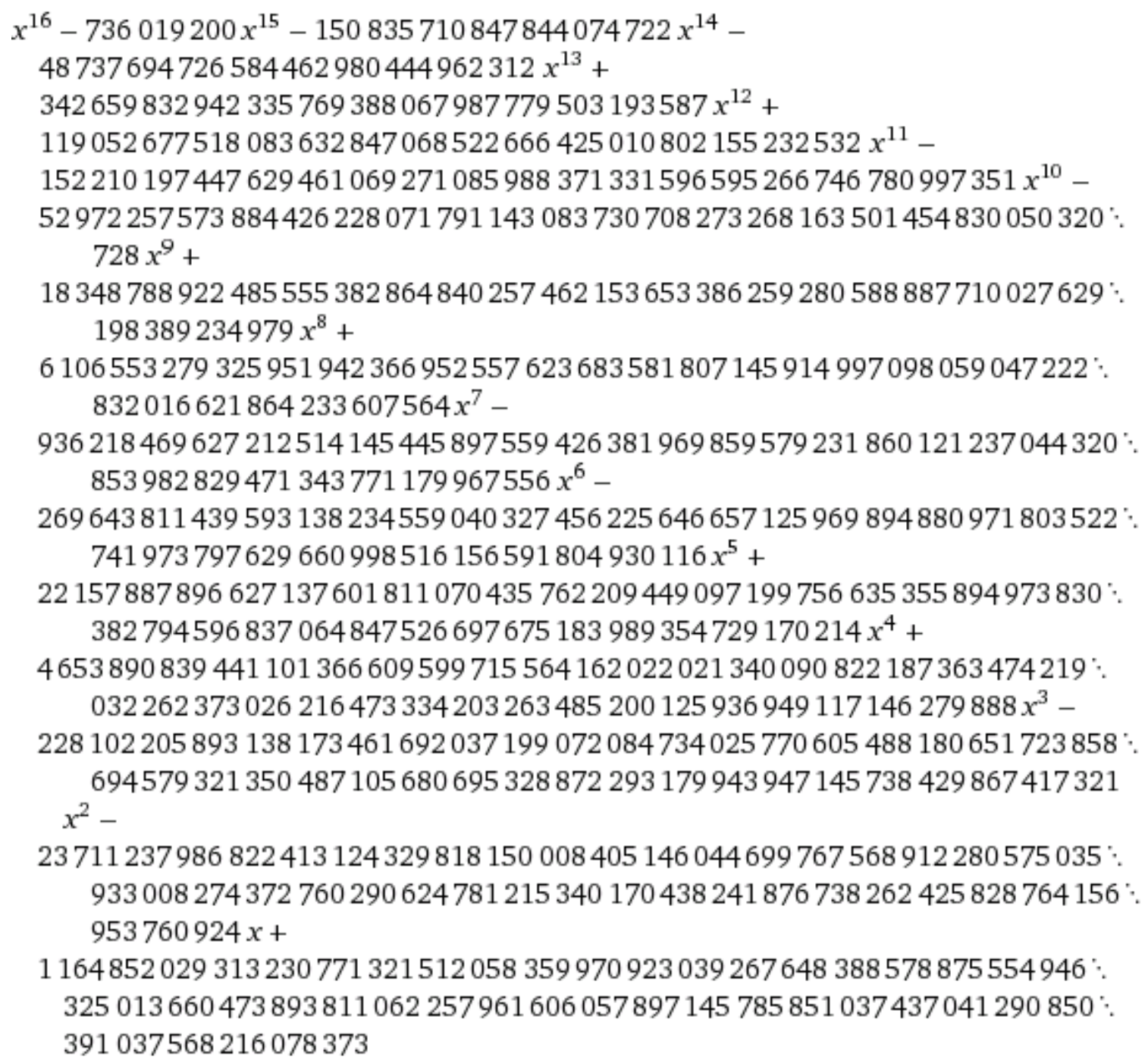

\section{General resource:}

http://www.wolfram.com/products/mathematica

\section{CONCLUSIONS}

The result of this research show that there is a matrix code for Solar System. Now we have the exact scientific proofs that there is an astronomical language that can be described by the theory of systems and cybernetics, and which functions in accordance with certain principles.

The process of sequencing in Solar System is conditioned and determined not only through astronomical, but also through cybernetic and information principles. The digital pictures of Solar System provide us with cybernetic and information interpretation of the 
scientific facts. Now we have the exact scientific proofs that there is a astronomic language that can be described by the theory of systems and cybernetics, and which functions in accordance with certain principles.

\section{References}

[1] L. Kurić, International Letters of Chemistry, Physics and Astronomy 10 (2014) 62-73.

[2] L. Kurić, J. Comput Sci Biol 2 (2009) 101-116.

[3] L. Kurić, Journal de la Societe de statistique de Paris 127(2) (1986).

[4] L. Kurić, The Insulin Bio Code - Zero Frenquencies, GJMR 10(1) (2010) 15.

[5] L. Kurić, Advances and Applications in Bioinformatics and Chemistry, 2010, 45-58.

[6] L. Kurić, GJMR 1(1) (2010) 15.

[7] L.Kurić, International Journal of Computer Technology and Application 2(2) (2011) 216-241.

[8] L. Kurić, International Journal of Computer Technology and Application 2(2) (2011) 258-273.

[9] L. Kurić, Journal of Chemical Enginerring and Material Science 2(5) (2011). 